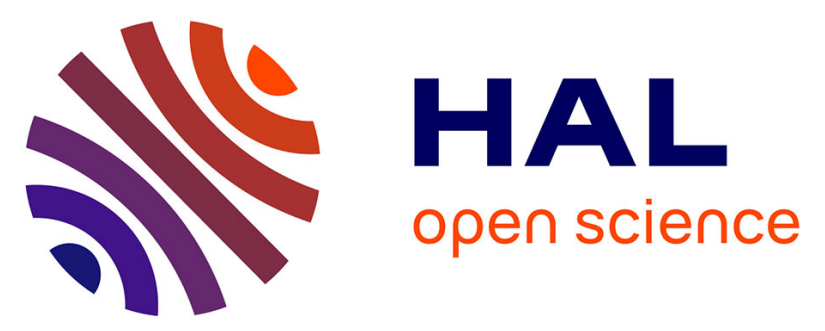

\title{
Inactivation of vimentin in satellite glial cells affects dorsal root ganglion intermediate filament expression and neuronal axon growth in vitro
}

Araksya Izmiryan, Zhenlin Li, Fatiha Nothias, Joel Eyer, Denise Paulin, Sylvia Soares, Zhigang Xue

\section{To cite this version:}

Araksya Izmiryan, Zhenlin Li, Fatiha Nothias, Joel Eyer, Denise Paulin, et al.. Inactivation of vimentin in satellite glial cells affects dorsal root ganglion intermediate filament expression and neuronal axon growth in vitro. Molecular and Cellular Neuroscience, 2021, 115, pp.103659. 10.1016/j.mcn.2021.103659 . hal-03368357

\section{HAL Id: hal-03368357 \\ https://hal.science/hal-03368357}

Submitted on 6 Oct 2021

HAL is a multi-disciplinary open access archive for the deposit and dissemination of scientific research documents, whether they are published or not. The documents may come from teaching and research institutions in France or abroad, or from public or private research centers.
L'archive ouverte pluridisciplinaire HAL, est destinée au dépôt et à la diffusion de documents scientifiques de niveau recherche, publiés ou non, émanant des établissements d'enseignement et de recherche français ou étrangers, des laboratoires publics ou privés. 
Inactivation of vimentin in satellite glial cells affects dorsal root ganglion intermediate filament expression and neuronal axon growth in vitro

Araksya Izmiryan ${ }^{1}$, Zhenlin Li $^{1}$, Fatiha Nothias ${ }^{2}$, Joel Eyer ${ }^{3}$, Denise Paulin ${ }^{1}$, Sylvia Soares ${ }^{2 \S}$, Zhigang $\mathrm{Xue}^{1 \S}$

1. Sorbonne Université, CNRS UMR8256, INSERM ERL U1164, Institut de Biologie Paris Seine, 75005 Paris, France

2. Sorbonne Université, CNRS UMR 8246, INSERM U 1130, Institut de Biologie Paris Seine, 75005 Paris, France

3. Laboratoire MINT, INSERM UMR 1066, CNRS UMR 6021, Université d'Angers, Institut de Biologie en Santé PBH-IRIS, 49033 Angers, France

\section{$\S$ Corresponding authors:}

Zhigang XUE : Sorbonne Université, CNRS UMR8256, INSERM ERL U1164, Institut de Biologie Paris Seine, 75005 Paris, France

E-mail : zhigang.xue@,sorbonne-universite.fr

Sylvia SOARES : Sorbonne Université, CNRS UMR 8246, INSERM U 1130, Institut de Biologie Paris Seine, 75005 Paris, France

E-mail : sylvia.soares@upmc.fr

\section{Highlights}

- Sciatic nerve injury alters neural IF protein synthesis in DRG neurons and SGCs

- Synemin M and peripherin synthesis is induced in axotomized large DRG neurons

- Vimentin is indispensable for GFAP upregulation in SGCs and neuronal IF remodeling

- Lack of vimentin and synemin enhances neurite initiation \& extension of regenerating DRG neurons 


\begin{abstract}
Peripheral nerve trauma and regeneration are complex events, and little is known concerning how occurrences in the distal stump affect the cell body's response to injury. Intermediate filament (IF) proteins underpin cellular architecture and take part in nerve cell proliferation, differentiation and axon regeneration, but their role in these processes is not yet fully understood. The present study aimed to investigate the regulation and interrelationship of major neural IFs in adult dorsal root ganglion (DRG) neurons and satellite glial cells (SGCs) following sciatic nerve injury. We demonstrated that the expression of neural IFs in DRG neurons and SGCs after axotomy depends on vimentin activity. In intact DRGs, synemin M and peripherin proteins are detected in small neurons while neurofilament L (NFL) and synemin L characterize large neurons. Both neuronal populations are surrounded by vimentin positive- and glial fibrillary acidic protein (GFAP)-negative SGCs. In response to axotomy, synemin $\mathrm{M}$ and peripherin were upregulated in large wild-type DRG neurons and, to a lesser extent, in vim-/- and synm-/- DRG neurons, suggesting the role for these IFs in axon regeneration. However, an increase in the number of NFL-positive small neurons was observed in vim-/- mice, accompanied by a decrease of peripherin-positive small neurons. These findings suggest that vimentin is required for injury-induced neuronal IF remodeling. We further show that vimentin is also indispensable for nerve injury-induced GFAP upregulation in perineuronal SGCs and that inactivation of vimentin and synemin appears to accelerate the rate of DRG neurite regeneration at early stages in vitro.
\end{abstract}

\title{
Graphical abstract
}

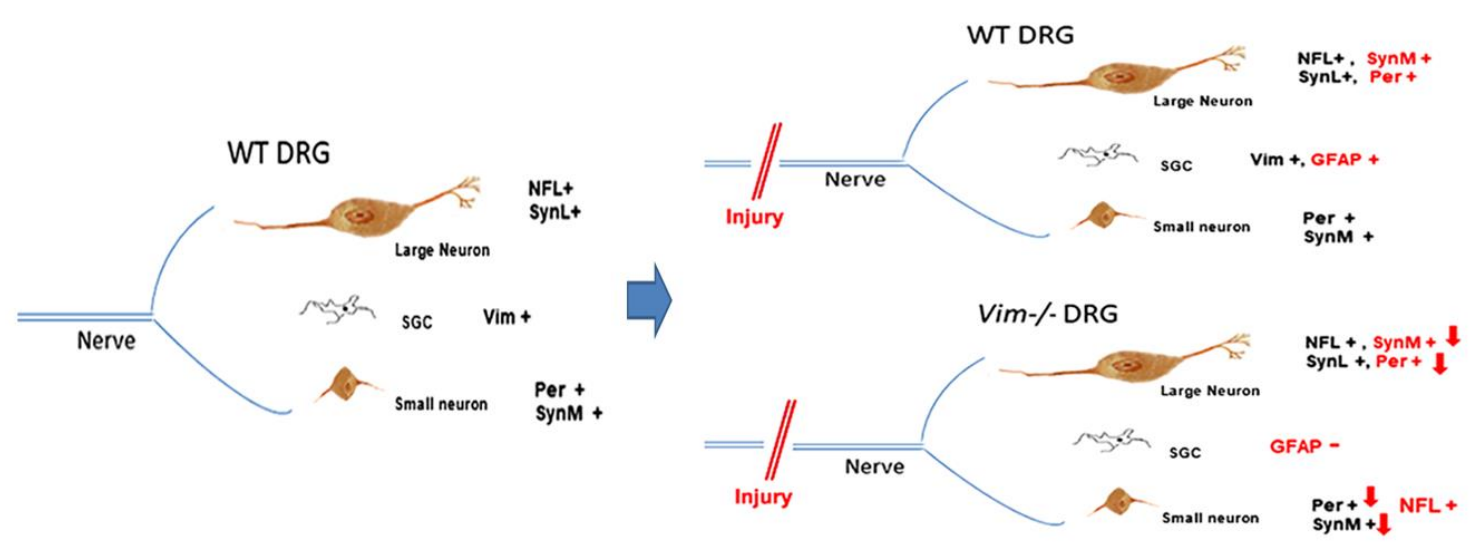


Keywords: nerve injury, GFAP, neurofilament, synemin, adult primary sensory neurons, axon regeneration

\section{Introduction}

Peripheral nerves are specialized organs that form a highly complex network throughout the neuronal body, providing the motor and/or sensory innervations to target organs. Histologically, peripheral nerves are composed of axons from neurons and the surrounding Schwann cells and satellite glial cells (SGCs). After peripheral nerve damage, these cells undergo extensive metabolic changes distal to the injury (Griffin and Hoffman, 1993), and axon regeneration is regulated through the coordinated spatiotemporal activation of multiple pathways. Indeed, as mainly shown in dorsal root ganglia (DRGs), which contain primary sensory neurons, several neuron-intrinsic molecular mechanisms contribute to the regenerative process, such as the retrograde transport of injury signals to the cell body of the neuron, leading to the expression of regeneration-related genes (Abe and Cavalli, 2008), local translation of mRNAs, axonal cytoskeleton reorganization, transport of materials, and the insertion of new membrane and cell surface molecules (Chandran et al., 2016; Vergara et al., 2018). However, whether the subsequent induction proceeds entirely within individual neurons with injured axons or involves the intermediary action of non-neural cells in the DRG is still not fully understood.

The neural cytoskeleton plays an irreplaceable role throughout regeneration by providing the basis of motility, structure, and stabilization of the newly formed axons, and axon regeneration involves pronounced changes in cytoskeletal gene expression that at least in part recapitulate the patterns observed in developing axons (Hoffman and Cleveland, 1988).

The cytoskeleton intermediate filaments (IFs) are composed of diverse proteins that are essential for cell architecture and functions, largely cell-type specific and are also major components of the neural 
cytoskeleton. IFs are also differentially regulated during axon regeneration as shown in DRG neurons for a down-regulation of neurofilaments and an upregulation of peripherin (Reid et al., 2010; Wong and Oblinger, 1990). It has also been reported that the inactivation of the IF GFAP gene, improves neuronal survival as well as neurite growth in mice during nerve regeneration by modifying adhesion molecule expression (Menet et al., 2001). Although the involvement of IFs in the process of nerve regeneration has been widely reported, few tend to focus on their regulation in neural cell bodies.

DRG neurons, derived from a common pool of neural crest cells, differentiate into a heterogeneous population of sensory neurons, which differ in many respects such as size, IF protein compositions, neuropeptides, neurotropic receptors, target field innervation and function. Based on morphological, ultrastructural and IF features, DRG neurons can be divided into two major neuronal subpopulations: large-light neurons with myelinated axons expressing high levels of neurofilament L (NFL) and smalldark neurons with unmyelinated axons that mainly express peripherin (Escurat et al., 1990; Goldstein et al., 1991; Sommer et al., 1985). In addition, our previous study has demonstrated that IF synemin isoforms also contribute to refining the characterization of mouse DRG neuron populations; synemin M is preferentially co-expressed with peripherin in small unmyelinated neurons, while synemin L colocalizes with NFL in large myelinated neurons (Izmiryan et al., 2009).

In peripheral sensory ganglia, SGCs have been shown to interact with sensory neurons and appear to be involved in the processing of afferent information (Pannese, 1964, 2010), but very little is known about their physiological and pharmacological properties as well as their correlation with IFs. Accumulating evidence suggests that SGCs are activated and undergo changes in cell number, structure and function in the sensory ganglia following peripheral nerve injury or inflammation, similarly to GFAP-expressing glial cells found in the central nervous system (CNS) (Cherkas et al., 2004; Hanani, 2005; Liang et al., 2010; Liu et al., 2012; Woodham et al., 1989). However, the regulation of these changes and their impact on signal transmission in sensory ganglia remains to be explored. 
The main objective of present study was to ascertain how the proteins of the different neural IFs of the peripheral nervous system (PNS) are regulated under traumatic situation. Accordingly, in vivo, we examined the dynamic synthesis of various neural IFs in DRG neurons of wild type (WT), vimentin knock-out (vim-/-) and synemin knock-out (synm-/-) mice during their axon regeneration following sciatic nerve injury. Particular attention was paid to the SGCs' response to nerve damage and its IF expression profile. Our experiments have shown for the first time that the sciatic nerve axotomy altered the synthesis of neural IF proteins in DRG neurons and that IF remodeling is dependent on the activity of vimentin during regeneration. To further elucidate the involvement of vimentin and synemin in axon growth in vitro, we analyzed neurite initiation and extension in vitro in culture of dissociated adult DRG neurons taken from vim-/-, synm-/- and WT mice.

\section{Materials and methods}

Animal

All experimental procedures on animals were done according to the European Community directive and the institutional guidelines (86/609/EEC; authorization number 91-78 to F.N.).

Generation of synm-/- and vim-/- mice has been described previously (Colucci-Guyon et al., 1994; Li et al., 2014); mice have been subsequently made congenic on an inbred C57BL/6 background. Animals were generated from our colony and genotyped by PCR analysis of tail genomic DNA, as described previously (Colucci-Guyon et al., 1994; Li et al., 2014). C57BL/6 wild type mice were used as control.

\section{Sciatic nerve transection}

Adult mice ( $n>6$, for each condition) were anesthetized with isoflurane. After skin incision, the right sciatic nerves were transected at the obturator tendon level, the nerve was replaced under the muscle and the incision was sutured by a single 9-0 through the epineurium to allow nerve regeneration. The left side (unoperated) nerve and corresponding DRG were used as controls, in addition to intact animals. 
After survival times of 3-, 5-, 8- and 30-days post-injury, animals were killed by cervical dislocation. The $4^{\text {th }}$ and $5^{\text {th }}$ lumbar (L4-L5) DRGs from both operated and unoperated sides were dissected out, frozen at $-140{ }^{\circ} \mathrm{C}$ and then kept at $-80{ }^{\circ} \mathrm{C}$ until sectioning. Sections (12 $\mu \mathrm{m}$ thickness) were cut on a cryostat and stored at $-80^{\circ} \mathrm{C}$ for immunocytochemistry detection.

\section{Cultures of dissociated adult DRGs neurons}

Dissociated adult DRG neurons from adult mice of different genotypes were cultured under the conditions described by Bouquet et al. (Bouquet et al., 2004). Cultures were fixed 12h and 24 hours after planting and neurites were immunocytochemically characterized by $\beta 3$ tubulin detection (Tuj1, Covance, 1:1500). Neurites of cultured DRG were visualized and photographed using a Zeiss Axiovert 200 inverted microscope equipped with an Axio Camera (Carl Zeiss SAS). For quantification, images were acquired on a macroscope Zeiss (Axiozoom V16) and analyzed using Fiji software (3 independent experiments, 30-50 neurons/genotype). Statistical significance was determined using t-test or ANOVA (Prism).

Immunohistochemistry study

DRG sections were treated for immunohistochemistry as described previously (Izmiryan et al., 2009; Nothias et al., 1995). The specific antibodies used were anti-synemin H/M (1:600), anti-synemin L (1:200) (Titeux et al., 2001; Xue et al., 2004), anti-NF-L (Chemicon, 1:500), anti-peripherin monoclonal $\mathrm{Ab}$ (Chemicon, 1:500), anti-vimentin (Progen Biothchnik, 1:200), anti-peripherin polyclonal Ab (1:300) (Escurat et al., 1990) and anti-glutamine synthetase monoclonal Ab (Millipore, 1:300). The secondary antibodies used were anti-mouse, or anti-rabbit immunoglobulins coupled to the Alexa 568 (1:500), Alexa 594 (1:500), Alexa 488 (1:500, Life Technologies) or Cy3 (Invitrogen, 1:500). Sections were then examined using Zeiss Axio and confocal microscopes. 
RT-PCR analysis

Left and right L4 and L5 DRGs were dissected as descripted above. Total RNA of isolated DRGs was extracted with Trizol Reagent (Invitrogen) and treated with RNase-free DNase (Qiagen), and was reversed transcribed using the miScript II RT Kit (Qiagen). The RT-PCR analysis was then performed with SYBR green PCR technology (Roche) as previously described (de Souza Martins et al., 2011; Izmiryan et al., 2009). Skeletal muscle RNA was used as a positive control. The predicted RT-PCR lengths of synemin $\mathrm{H}$ and $\mathrm{M}$ are $1452 \mathrm{bp}$ and 546bp, respectively. The following primers were used for PCR:

Synemin H or M: Forward 5'-AGTCAGGGAGCGTTTCTGTGGACG-3'

and Reverse 5'-ATCGCTCTCGTGTCGCTCAAATCC-3'

GAPDH: Forward 5'-AGTCCATGCCATCACTGCCACCCA-3' and Reverse 5'-TCCACCACCCTGTTGCTGTAGCCG-3'

Western blot assay

Total protein was extracted from frozen lungs of mice. Lungs were homogenized using a Kinexus lysis buffer (mmol/L: MOPS, 20 pH 7.2; EGTA, 2; EDTA, 5; NaF, 30; glycerophosphate, 40; sodium pyrophosphate, 20; NaVO4, 1; PMSF, 1; containing 0.5\% Nonidet P40, and protease inhibitors cocktail (Sigma)). Extracts were centrifuged at $14000 \mathrm{rpm}$ for $20 \mathrm{~min}$ at $4^{\circ} \mathrm{C}$ and the protein concentration in the supernatant was determined by the Bradford method (Bio-Rad). Samples $(20 \mu \mathrm{g})$ were separated by 4-15\% gradient polyacrylamide gel and transferred to nitrocellulose membrane (Bio-Rad). Proteins were detected with anti-vimentin (Abcam 1:2000), anti-synemin (1:2000) (Li et al., 2014) and antiHSP60 (Santa-Cruz, 1/1000) as previously described (Galmiche et al., 2013).

Statistical analysis of in vivo imaging 
The statistical significance of quantitative variables of spinal ganglion neurons was performed by twoway factorial ANOVA. Tukey tests were used for comparison of specific experimental groups. For all analyses, two-tailed $P$-values of $<0.05$ were considered statistically significant. Values are presented as means \pm SEM.

At least six mice per conditions and ten sections per animal (at least five sections to cover an entire ganglion) were used for the statistical analysis.

\section{Results}

\section{Modification of IF protein distribution in wild type DRG neurons during axon regeneration}

In the present study, experiments were carried out using two anti-synemin antibodies as markers (Titeux et al., 2001; Xue et al., 2004). The anti-synemin L antibody specifically recognizes the L isoform of synemin whereas the anti-synemin $\mathrm{H} / \mathrm{M}$ antibody recognizes both $\mathrm{M}$ and $\mathrm{H}$ synemin isoforms. In order to determine the expression levels of synemin $\mathrm{H}$ and $\mathrm{M}$ isoforms, we investigated their expression at the mRNA level in intact adult L4 and L5 DRGs as well as during axon regeneration following sciatic nerve injury. RT-PCR analysis clearly demonstrated that the synemin H isoform was expressed neither in intact adult DRG nor during axon regeneration. However, synemin M mRNA was detected in all conditions and, interestingly, its levels increased after sciatic nerve injury, with the highest expression level detected in DRGs at 8 days after injury (Figure 1U). Through immunostaining and data collection, we conclude that the anti-synemin H/M antibody specifically and solely recognizes the synemin $\mathrm{M}$ isoform in adult DRGs.

After mouse genotyping, to further confirm the three genotype mice used in our experiments, we performed a Western blot analysis with the lungs of the mice, showing the presence of vimentin and synemin proteins in control mice (WT), but not in vim-/- and synm-/- mice, respectively (Figure $1 \mathrm{~V}$ ). In intact (control) DRGs, we found two subpopulations of neurons, each expressing a unique type of 
synemin isoform. Small neurons (consisting of neurons 15 to $30 \mu \mathrm{m}$ in diameter) (Ferri et al., 1990; Sommer et al., 1985) contain synemin M and peripherin proteins, but lack NFL and synemin L proteins (Figures $1 \mathrm{~A}-\mathrm{E})$. In the second population corresponding to large size neurons (includes neurons with a diameter ranging from 30 to $60 \mu \mathrm{m}$ ) (Ferri et al., 1990; Sommer et al., 1985), synemin M and peripherin were absent, while the co-labeling of synemin L and NFL was observed (Figures 1A, 1C-E). To define the time course and the magnitude of change of the synemin content in the DRG neurons during axonal regeneration, L4 and L5 DRG sections were examined at 3-, 8-, and 30 days following sciatic nerve injury through immunostaining for various IFs.

Based on our observations, we identified a significant change in the distribution of IF proteins in large DRG neurons of WT mice during regeneration. An induction of peripherin and its partner, synemin M, was observed in large neurons starting from 3 days after nerve injury, concomitantly with NFL and synemin L (Figures 1F-G, I-L, N-Q and S-T; 4A, 4C, 4E and 4G). No apparent modifications were found in small DRG neurons of WT mice, which were positive for synemin M and peripherin (Figures 1F-G, I-L, N-Q and S-T; 4B and 4D). Therefore, during axon regeneration, synemin $\mathrm{M}$ and peripherin expression is upregulated in large DRG neurons. The expression of NFL and synemin L proteins in large neurons (Figures 1F, 1H-K, 1M-P and 1R-T, 4E and 4G) has not been perturbed by injury. However, a decrease of approximately $35 \%$ from the total number of neurons (large + small) was observed (data not showed), which confirmed earlier reported data (McKay Hart et al., 2002; Raginov and Chelyshev Iu, 2003). The presence of synemin M and peripherin in both neuronal populations was detected in almost all neurons during the first 8 days of regeneration. This finding indicates that synemin and peripherin actively participate in all DRG sensory neuron populations during axon regeneration. Interestingly, at later stages of this regenerative period, the initial expression pattern of synemin $\mathrm{M}$ and peripherin recovered progressively (close to the intact DRG), as was observed at 30 days post-injury (Figures 1Q, S-T; 4A and 4C). In addition, the increase in synemin M mRNA found by RT-PCR analysis at 8 days post-injury (Figure 1U) may account for its upregulation in large DRG 
neurons.

We subsequently analyzed the expression pattern of IFs in satellite glial cells (SGCs) as well as changes during axon regeneration. In the DRG of intact adult WT mice, a ring-like pattern of vimentin was detected in SGCs surrounding all sensory neurons (5A). During the first week following sciatic nerve transection, analyzed here at 5 days post-injury, vimentin immunohistochemistry was still detected around both small and large DRG neurons. (Figures 2B-C, E-F, H-I and K-L). In contrast, all DRG sensory neurons were positive for synemin proteins (Figures 2A, C-D, F-G, 2I-J and L), but negative to vimentin (Figures 2G-L). No synemin-positive SGCs were found in DRG sections (Figures 2G-L). The location of the vimentin labeling was visibly cytoplasmic and close to the outer membrane, which proved of interest considering the widespread acceptance of vimentin's role in regulating the communication between the cell surface and the nucleus (Sugimura et al., 1989). Furthermore, the SGC population surrounding large neurons exhibited intense immunoreactivity whereas those surrounding small neurons were weakly labeled (Figure 2G-L). This observed phenomenon is most likely related to the higher number of SGCs surrounding large DRG neurons compared to those of smaller size. It should also be noted that vimentin staining was present in areas solely composed of fibers, which could be attributed to its expression by Schwann cells (Figures 2B and 2E).

\section{Change of NFL and peripherin expression in the DRG neurons of vimentin knock-out mice during}

\section{axonal regeneration}

In order to investigate whether IF regulation in intact and regenerating DRG neurons would be impacted by changes in IF expression of DRGs, vim-/- mice were employed to explore potential outcomes. In intact vim-/- adult DRGs, slight changes in the distribution of neuronal IF proteins were observed compared to WT mice: several small neurons were positive for synemin L (Figures 3C, 4H) and NFL (Figure 4F), and a few large neurons positive for synemin M (Figures 3A, 4C). No obvious changes were found in the expression patterns of peripherin proteins (Figures 3C, 4A-B). Following 
peripheral nerve injury, the distribution of neuronal IF proteins in vim-/- mice was different from that in WT mice. As in WT DRGs, peripherin and synemin M proteins were upregulated in large vim-/- DRG neurons after axotomy while NFL remained expressed (Figures 3D-L, 4A, 4C and 4E). However, in contrast to WT DRGs, this upregulation was not detected in all large vim-/- DRG neurons during axon regeneration at 8 days post-injury, the time point corresponding to detection of peripherin and synemin $\mathrm{M}$ in all DRG of WT mice. Therefore, only 60-70\% of large neurons simultaneously expressed peripherin, synemin M and NFL proteins between day 3 and day 8 (Figures 3D, 3G, 4A, 4C and 4E). Large vim-/- DRG neurons only exhibited a slight decrease in synemin L immunostaining (Figure 4G) compared to NFL-positive neurons whose number was similar to that of WT mice (Figure 4E). Among the small DRG neurons, a significant decrease in peripherin expression was noted in vim-/- DRGs during the first 8 days of regeneration (Figure 4B), but the same was not observed for synemin $\mathrm{M}$ (Figure 4D). Surprisingly, following sciatic nerve injury, the number of NFL-positive small neurons increased rapidly, reaching a peak on day 8 of regeneration in vim-/- mice (approximately $70 \%$ at day 8 , Figures 3D, 3J and 4F). However, this significant change did not occur in WT mice (Figures 1 and 4F). Furthermore, the increase in NFL-positive small neurons was accompanied by a reduction in the number of peripherin-positive small neurons (Figure 4B). On the other hand, compared to WT mice, the number of small DRG neurons expressing synemin L in vim-/- mice was also increased, but to a lesser extent (Figure 4H). These results demonstrate that, at least in the early stages of DRG axon regeneration, peripherin, synemin, and NFL are closely related to vimentin expression.

\section{IF protein expression in synemin knock-out DRG neurons following nerve injury}

The change observed in WT DRG regarding IF protein expression during the axon regeneration was also detected in synm-/- mice; an induction of peripherin synthesis in large neurons post-injury, but at a lesser extent (Figures 3N-P, 4A). Only 60-70\% of large neurons expressed both peripherin and NFL during the first 8 days of regeneration (Figure $3 \mathrm{~N}-\mathrm{O}, 4 \mathrm{~A}$ ). The absence of synemin did not affect the 
expression of peripherin and NFL proteins in the small neurons during regeneration (Figures 3N-P, 4B and 4F). This phenomenon indicated that the expression of peripherin in small DRG neurons is independent of the presence of synemin. After one month post-regeneration, approximately $20 \%$ of large DRG neurons in synm-/- mice continued to synthesize peripherin proteins similarly to WT mice (Figures 3P, 4A). No modification in the synthesis of NFL was observed in large neurons (Figures 3NP, 4E). Notably, the total number of DRG neurons was decreased by approximately $30 \%$ after axotomy in synm-/- mice (data not shown).

\section{Vimentin expression in SGCs is required to maintain expression of IFs in DRG during their axon}

\section{regeneration}

Injury to the peripheral nerve is known to induce a vigorous regeneration process, which, under normal conditions, leads to the repair and eventual recovery of function in the peripheral nervous system. Current information indicates that glial cells participate in all normal and pathological processes of the CNS. Although much less is known about SGCs in sensory ganglia, it appears that these cells share many characteristics with their central counterparts. Our analysis showed that no GFAP protein was detected in intact adult DRGs in all three mouse models examined (WT, synm-/- and vim-/-; Figures 5A-C). However, a novel synthesis of GFAP was induced in SGCs following sciatic nerve injury in WT and synm-/- mice (Figures 5D-E, 5G-H). No significant preferential expression of GFAP surrounding small or large neurons was observed. In contrast, no GFAP protein was detected in vim-/mice, at least during the first 8 days of regeneration (Figures 5F and 5I). To determine whether the lack of GFAP protein was a direct consequence of vimentin absence or due to abnormal development of SGCs resulting from the deletion of vimentin genes, we investigated the expression of glutamine synthase (GS), a known SGC marker, in DRG following sciatic nerve injury. No apparent changes in GS synthesis were detected by our experiments both before and after DRG axotomy in SGCs of vim-/mice as well as in WT and synm-/- mice (Figures 5J-O). This result clearly indicates that in the absence 
of vimentin, although the state of SGCs was not affected, GFAP expression is compromised during DRG axon regeneration.

\section{Synemin and vimentin are involved in neurite initiation of adult regenerative DRG neurons}

We next investigated whether knockdown of vimentin or synemin genes impacts adult DRG neurite regeneration, 12 and 24 hours after planting neurons in vitro, reflecting initiation and extension states, respectively. After 12 hours of culture, less than 2\% of WT neurons exhibit regenerated neurites. However a significant increase (3.13 folds as high) in the number of synm-/- DRG neurons with neurites was observed compared to WT DRG neurons (Figure 6A). The number of vim-/- neurons exhibiting neurites was also increased compared to WT neurons (2.52 folds as high), although not significant. Several neurites were able to growth from the same cell body; some appeared highly branched (Figure 6B), however, no specific morphology could be attributed to one genotype. Quantification of total neurite length (Figure 6A) showed that WT and vim-/- neurons had the same rate of neurites extension (218 and $358 \mu \mathrm{m}$, respectively). Synm-/- neurons had a significantly greater extension capacity $(540 \mu \mathrm{m})$ than the other two genotypes (Figure 6B). Thus, synemin appears to at least slow the rate of neurite growth. After 24 hours in culture, around $28 \%$ of WT neurons were able to extend their neurites. No difference in the percentage of neurons bearing neurites was observed between genotypes (Figure 6C). However, at 24 hours, the total neurite length of vim-/- and synm-/neurons was longer than that of WT (Figure 6B-C). The results of quantifying the longest neurite of neurons of different genotypes are in the same direction as the quantifications of total neurite length. At 12 hours, the longest neurite of synm-/- neurons was significantly longer than that of WT and vim-/neurons. However, the neurite extension rates of WT and vim-/- neurons were similar. Notably, after 24 hours in vitro, the longest neurite of both vim-/- and synm-/- neurons was longer than that of WT neurons (Figure 6D). Altogether, the lack of vimentin or synemin appears to accelerate the rate of axonal growth in vitro at an early stage. 


\section{Discussion}

In the present study, we first demonstrated that the expression of DRG neuronal IFs following sciatic nerve axotomy is dependent on vimentin activity in SGCs. In the absence of vimentin, the expression of peripherin and synemin M in axotomized large DRG neurons, and NFL and synemin L in small neurons are impacted. Second, GFAP protein synthesis is upregulated in SGCs after nerve injury, but does not occur in vim-/- SGCs, showing that vimentin is essential for GFAP upregulation in perineuronal SGCs. Finally, we have shown that inactivation of vimentin and synemin appears to accelerate the initiation of DRG neurite regeneration and improve neurite extension capacity.

The sensory ganglia are composed of morphological and functionally distinct neurons as well as of glial cells that surround these neurons. It is commonly known that within the ganglia, sensory neurons do not form neither electrical nor chemical synapses, as each soma is surrounded by glial cells, known as SGCs. Perineuronal SGCs have an intimate anatomical relationship with sensory neurons that suggests close functional interaction and mutual support (Fields and Stevens-Graham, 2002; Hanani, 2005). IFs are an essential component of the cytoskeleton of neurons and glial cells. In order to understand the changes and regulation of different neural IF proteins during trauma, we examined several aspects of this relationship in adult sensory DRGs after nerve injury. The findings of the present study demonstrate that the response of sensory neurons to nerve injury involves local changes not only in neural IFs, but also a series of alterations in perineuronal SGCs. In response to axotomy, peripherin and synemin M were upregulated in large DRG neurons, but to a lesser extent in vim-/- and synm-/mice. DRG large neurons are known to have myelinated axons (Goldstein et al., 1991; Sommer et al., 1985), which do not synthesize peripherin and synemin $M$ in the intact state. Our result showed that new IF synthesis in large DRG neurons was affected by the inactivation of vimentin and synemin during axon regeneration. Synemin is an atypical IF whose complex expression patterns and functions have remained elusive until now. Our previous studies showed that synemin $M$ is present in 
undifferentiated embryonic stem (ES) cells as early as the pluripotency factor Oct-4 and keratin 8 and predated nestin. Knockdown of synemin in ES cells has no effect on the expression of Oct4, Nanog and SOX2, but reduces the expression of another cytoskeleton IF member, keratin 8 (de Souza Martins et al., 2011). However, the relationship between synemin, vimentin and peripherin during neuronal IF remolding remains to be explored. In comparison, small DRG neurons are unmyelinated neurons that synthesized peripherin and synemin $M$ in the intact state, and the different impacts of vimentin and synemin $\mathrm{M}$ on the regulation of peripherin expression were observed in these neurons after injury (Figure 4B). The peripherin expression was unaffected by synemin deletion, suggesting that peripherin expression in small DRG neurons is independent of the presence of synemin. This result confirms that synemin acts more as an IF-associated protein in the small DRG neurons, which cannot organize itself into a cytoplasmic network but must bind to preexisting filaments to form a network (Granger and Lazarides, 1980; Green et al., 2005). In contrast, a significant decrease in peripherin expression was noted among small regenerating vim-/- DRG neurons after sciatic nerve injury. Peripherin is a less studied type III IF class to date, and little is known about the regulation of its expression. Our result suggests that peripherin expression in small DRG neurons correlates with vimentin expression in SGCs. Furthermore, the nature and extent of roles played in neurite outgrowth by the major neuronal IFs are still not fully understood. After a CNS injury, the absence of GFAP and vimentin changes the structure of astrocytes (Gimenez et al., 2000), which are unable to subsequently form IF filamentous networks (Pekny et al., 1999). But the absence of vimentin does not alter the astroglial response to neurological insult (Menet et al., 2003; Pekny, 2001). It has also been reported that the fraction of vesicles with directional mobility was reduced in mouse astrocytes deficient in GFAP and vimentin (Potokar et al., 2007). The results of our in vitro culture experiment of dissociated DRG neurons from uninjured vim-/and synm-/- mice showed that the absence of vimentin or synemin appeared to accelerate the initiation and extension of axonal regeneration, at least at early stages (Figure 6). Similar results have been reported for the inactivation of GFAP gene in astrocytes, which improves neuronal survival and neurite 
growth in vitro (Menet et al., 2001). However, reduced neurite growth has also been reported in culture in vitro of DRG neurons from mice lacking vimentin after conditioning sciatic nerve lesion (Perlson et al., 2005). The reasons for this discrepancy are not yet clear and may be related to sciatic nerve injury prior to culturing DRG neurons, the approaches used to culture the neurons, and the culture conditions. Peripheral nerve injuries are known to induce a vigorous regeneration process. Our current experiments have shown that the sciatic nerve axotomy can alter the synthesis of neural IF proteins in DRG and that DRG IF remodeling depends on the expression of vimentin in SGC. However, it remains to be determined whether axotomy can alter the effect of IFs on axonal growth during neuritogenesis. Nevertheless, all results indicate that IF proteins directly or indirectly influence neuronal regeneration processes.

Glial cells in the PNS and CNS have long been recognized for their mechanical and metabolic responses to injury characterized by tightly-regulated control over the neuronal microenvironment through the activation of SGCs and by astrocytosis (Shinder et al., 1998) respectively, which facilitates the maintenance of neuronal homeostasis (Hanani, 2005). Perineuronal glial cells in DRGs, similarly to Schwann cells, express the IF protein vimentin (Nascimento et al., 2008; Vega et al., 1989). Following neuronal damage, the expression of various IFs resumes or is modified; vimentin and GFAP are reupregulated in proliferating glial cells (Gillen et al., 1995; Oblinger and Lasek, 1988; Watkins and Maier, 2002) and become the main cytoskeletal proteins of these cells (Ramer et al., 2000) while the expression of mature IFs such as NF-L and NF-H decreases in damaged neurons (Gillen et al., 1995; Oblinger and Lasek, 1988; Ramer et al., 2000; Willis et al., 2005). However, dynamic changes occurring through the modulation of the cytoskeleton in axon and ganglion cells constitute a key pathway for neural regeneration (Lariviere and Julien, 2004), whose exact mechanism is not yet completely established. A molecular exchange from neuron to perineuronal SGCs has been demonstrated by a retrograde trace method using a fluorochrome diamidino (Christie et al., 2015). This interaction is ongoing and bilateral. It recently reported that SGCs can activate peroxisome proliferator- 
activated receptor alpha (PPAR alpha), a ligand-activated nuclear receptor with the unique ability to bind lipid signaling molecules, to promote axon regeneration in adult peripheral neurons (Avraham et al., 2020). In the present work, although a vimentin deficiency did not prevent the expression of synemin and peripherin proteins in sensory neurons, the percentage of large DRG neurons expressing peripherin, synemin $\mathrm{M}$ and $\mathrm{L}$ was decreased following neurological insult. However, we also observed an upregulation of NFL-positive small neurons following nerve damage solely in vimentin-deficient mice as well as a decrease in peripheral protein-positive small neurons. These changes in expression levels did not occur in WT mice. In the meantime, the regenerative GFAP expression in SGCs associated with nerve injury was completely blocked when vimentin gene was deleted. Using cultures of dissociated adult DRG neurons, we demonstrate that, although expressed only in SGC cells, vimentin appears to have a rate-limiting effect on initial stages of axonal regeneration, as neurite extension is increased in vim-/- neurons. Taken together, these results illustrate the robust interaction between intraganglionic neurons and SGCs, while also demonstrating how the presence of vimentin is indispensable for injury-induced neuronal IF remodeling as well as for GFAP upregulation in SGCs in response to axon damage. These findings further suggest that the regenerative response of SGCs to the distal axon lesion of its neighboring neurons could directly affect injury-induced neuronal IF remodeling. This raises questions pertaining to the mechanism linking axonal damage to changes in IF expression in ganglion cells as well as to the events leading to interactions between injured sensory neurons and surrounding SGCs. Detailed molecular models of these processes will ultimately help in improving understandings regarding the effects of IF activity in glia-neuron communication, and its role in physiological and pathological changes of ganglia. 


\section{Acknowledgments}

We thank Dr. A. Parlakian and M. Gublin for histology assistance, and Sareen Kardjian for linguistic improvements.

\section{Grant information}

This work was supported with grants from the Association Française contre les Myopathies (AFM) (contract $\left.\mathrm{n}^{\circ} 13607,14848\right)$ and the Foundation pour la Recherche Médicale (FRM). AI held a fellowship from the French government.

\section{Conflict of Interest Statement}

The authors declare no competing interests.

\section{Statement of author contributions:}

ZX designed research, AI, ZX, ZL, SS, FN performed research, ZX, ZL, DP contributed to data analysis, ZX, FN, ZL, SS, JE for critical revision of the manuscript, ZX, ZL, FN, SS wrote the paper. 


\section{Legends}

Figure 1: The evolution of synemin, peripherin and neurofilament L proteins during DRG regeneration in WT mice (A-T). In intact animal models, the different associations of synemin M (SynM) and L (SynL) with peripherin (Per) and neurofilament L (NFL) define two distinct neuron subpopulations of DRG (A-E). Synemin M has a preferential interaction with peripherin in small neurons (A-B, D-E), while synemin $\mathrm{L}$ is colocalized with NF-L in large neurons (C-E). An induction of peripherin and synemin $\mathrm{M}$ in large neurons, colocalized with existing NFL and synemin L (F-G, I-L, N-Q, S-T), was observed starting at day 3 of regeneration, while almost no change in IF proteins was detected in small neurons (F-G, I-L, N-Q, S-T). No disturbance of NFL and synemin L protein expression in large neurons due to nerve injury was observed (F, H-K, M-P, R-T). Scale bar $20 \mu \mathrm{m}$ for A-D, F-I, K-N, P-S and $150 \mu \mathrm{m}$ for $\mathrm{E}, \mathrm{J}, \mathrm{O}, \mathrm{T}$.

Expression of synemin $\mathrm{H}$ and $\mathrm{M}$ mRNA in intact $\mathrm{DRG}(\mathrm{Ct})$ and $\mathrm{DRG}$ at 3, 8 and 30 days following nerve injury analyzed by RT-PCR (U) showed that only synemin M (SynM) mRNA was expressed in WT DRG and during DRG regeneration, while synemin $\mathrm{H}(\mathrm{SynH})$ was never detected. Skeletal muscle $(\mathrm{SkM})$ was used as a positive control, as described in the Methods.

Western blot analysis of lungs from vim-/-, synm-/- and control mice (V) showed the presence of vimentin (Vim) and synemin M (SynM) in control mice (WT), but not in vim-/- (V-/-) and synm-/- (S-/-) mice, respectively. HSP60 was used as a loading control.

Figure 2: Immunodetection of synemin and vimentin in DRG 5 days after sciatic nerve transection in WT mice.

Immunostaining showed vimentin expression in satellite glial cells (SGCs) around the DRG neurons (B, $\mathrm{E}, \mathrm{H}$, and $\mathrm{K}$ ). Almost all SGCs were stained by an anti-vimentin antibody. The DRG neurons were positive to synemin $\mathrm{M}(\mathrm{SynM})(\mathrm{A}, \mathrm{G})$ and $\mathrm{L}(\mathrm{SynL})(\mathrm{D}, \mathrm{J})$. No synemin expression was detected in SGCs (A-L). The SGC population surrounding large neurons exhibited more intense vimentin(Vim) labeling in comparison to small neurons (G-L, arrows indicate the small neurons). Scale bar $200 \mu \mathrm{m}$ for A-F and $20 \mu \mathrm{m}$ for G-L.

Figure 3: The change of IF proteins during DRG regeneration in vim-/- and synm-/- mice. Panels A-C demonstrate the expression of synemin M (SynM), synemin L (SynL), NFL and peripherin (Per) in the intact control vim-/- mice.

Panels D-L show changes in IF protein in vim-/- mice at days 3-, 8- and 30- following sciatic nerve injury. In absence of vimentin, the expression of synemin $\mathrm{M}$ and peripherin was observed in both large and small DRG neuron populations (D-L). Several large neurons did not simultaneously express synemin M and NFL proteins (D, G, arrow). A novel synthesis of the NFL protein was also detected in small neurons (D, J, arrowhead).

Panels M-P show peripherin and NFL immunostaining in DRG of synm-/- mice. Panel M illustrates the lack of peripherin (Per) induction in the intact control synm-/- large DRG neurons. Panels N-P show peripherin and NFL expression at days 3-, 8- and 30- following sciatic nerve injury. The absence of synemin did not modify the tendency of the novel peripherin synthesis in large DRG neurons. However, not all large neurons expressed both peripherin and NFL during the first 8 days of regeneration (N-P). The asterisks indicate the colocalization of peripherin and NFL in large neurons. Scale Bar $=20 \mu \mathrm{m}$.

Figure 4: Statistical analysis of DRG neurons that expressed different neural IF proteins during regeneration in wild type (WT), synm-/- and vim-/- mice. The novel synthesis of peripherin (Per) and synemin M (SynM) was observed in large neurons (LN) during DRG regeneration in three mouse models (A and $\mathrm{C}$ ). A decrease in the number of large neurons stained by anti-peripherin was observed in both synm-/- and vim-/- mice at days 3- and 8- post injury. In comparison to WT mice (A), a similar 
decrease in the number of synemin M-positive large neurons was found in vim-/- mice (C). For small neuron (SN) subpopulations, no variations in the number of neurons positive for peripherin was observed in WT and synm-/- mice (B), but a decreased in number of peripherin-positive small neurons was detected during the first 8 days of regeneration in vim-/- mice (B). No variations in the number of synemin M-positive neurons were observed in WT and vim-/- mice (D). Following sciatic nerve injury, the synthesis of NFL in large neurons of all three types of mice remained unchanged (E), but the number of small DRG neurons that synthesized NFL increased rapidly in vim-/- mice only, increasing by approximately $70 \%$ on day 8, and was not observed in WT and synm-/- mice (F). Following peripheral nerve damage, a decrease in the number of synemin $\mathrm{L}$ (SynL) positive large neurons was observed in vim-/- mice (G), while a small number of small neurons were also positive to synemin $\mathrm{L}$ in WT and vim-/- mice $(\mathrm{H})$.

Two-way factorial ANOVA for independent samples was used for all panels. Data are means \pm SEM. $\mathrm{n}>12$. Asterisks indicate statistically significant differences versus WT group: $* * * \mathrm{p} \leq 0.001$.

Figure 5: The immunodetection of IF proteins and glutamine synthase (GS) during DRG regeneration in WT, synm-/- and vim-/- mice.

Vimentin (Vim) protein expression in SGCs was detected in WT and synm-/- control mice (A-B), while no GFAP-positive SGCs in the three types of intact mice (control) (A-C) occurred. During regeneration, a novel expression of GFAP was detected in SGCs of the injured DRG at 3 days (D-E) and 8 days (G$\mathrm{H}$ ) in the WT and synm-/- mice, but no SGCs were stained by anti-GFAP in vim-/- mice (F and I). In contrast, the synthesis of glutamine synthase (GS) in SGCs was maintained in these three mouse models $(\mathrm{J}-\mathrm{O})$. Scale bar $=20 \mu \mathrm{M}$.

Figure 6: Comparison of outgrowth of adult DRG neurons from different genotype mice in vitro.

A: Quantification of neurite regeneration of WT, vim-/- and synm-/- neurons after $12 \mathrm{~h}$ in vitro. Left panel: percentage of neurons bearing neurites. Values of WT neurons were normalized and statistical analysis was performed for vim-/- and synm-/- neurons compared to WT (t-test. * $\mathrm{p}=0.04$ ). Right panel: total neurite length. No significant difference in total neurite length was observed between WT and vim-/- neurons. In contrast, the total neurite length of synm-/- neurons was longer than that of WT and vim-/- neurons. (Anova Turkey's test, ** WT vs synm-/- $\mathrm{p}=0.0011$, ** vim-/- vs synm-/- $\mathrm{p}=0.0027$ ).

B: Immunocytochemical characterization of cultured DRG neurons from WT, vim-/- and synm-/- mice, after 12 and 24 hours in vitro. Neurons were immunocytochemically characterized by b-III-tubulin detection. Note the increase in neurite growth between 12 and 24 hours in vitro. At 24 hours, total neurite extension was more robust in vim-/- and synm-/- neurons compared to WT neurons.

C: Quantification of neurite regeneration of WT, vim-/- and synm-/- neurons after 24 hours in vitro. Left panel: percentage of neurons bearing neurites. Values of WT neurons were normalized and statistical analysis was performed for vim-/- and synm-/- neurons compared to WT (t-test, not significant). Right panel: total neurite length. Statistical analysis showed that the total neurite length of vim-/- and synm-/- neurons was longer than that of WT neurons. (Anova Turkey's test, ** $\mathrm{p}=0.0095$, $* * * * \mathrm{p}<0,0001)$.

D: Quantification of the longest neurite of WT, vim-/- and synm-/- neurons after 12 hours and 24 hours in vitro. At 12 hours, no significant difference was observed between WT and vim-/- neurons. In contrast, the longest neurite of synm-/- neurons was significantly longer than that of WT and vim-/neurons. Note that after 24 hours in vitro, the longest neurite of vim-/- and synm-/- neurons was longer than that of WT neurons. (Anova Turkey's test; at 12 hours: *** wt vs synm-/- $\mathrm{p}=0.0001$, ***vim-/- vs synm-/- $\mathrm{p}=0.0008$; at 24 hours: $* * * * \mathrm{p}<0.0001)$. 


\section{References}

Abe, N., Cavalli, V., 2008. Nerve injury signaling. Curr Opin Neurobiol 18, 276-283.

Avraham, O., Deng, P.Y., Jones, S., Kuruvilla, R., Semenkovich, C.F., Klyachko, V.A., Cavalli, V., 2020. Satellite glial cells promote regenerative growth in sensory neurons. Nat Commun 11, 4891.

Bouquet, C., Soares, S., von Boxberg, Y., Ravaille-Veron, M., Propst, F., Nothias, F., 2004. Microtubule-associated protein $1 \mathrm{~B}$ controls directionality of growth cone migration and axonal branching in regeneration of adult dorsal root ganglia neurons. The Journal of neuroscience : the official journal of the Society for Neuroscience 24, 7204-7213.

Chandran, V., Coppola, G., Nawabi, H., Omura, T., Versano, R., Huebner, E.A., Zhang, A., Costigan, M., Yekkirala, A., Barrett, L., Blesch, A., Michaelevski, I., Davis-Turak, J., Gao, F., Langfelder, P., Horvath, S., He, Z., Benowitz, L., Fainzilber, M., Tuszynski, M., Woolf, C.J., Geschwind, D.H., 2016. A Systems-Level Analysis of the Peripheral Nerve Intrinsic Axonal Growth Program. Neuron 89, 956970.

Cherkas, P.S., Huang, T.Y., Pannicke, T., Tal, M., Reichenbach, A., Hanani, M., 2004. The effects of axotomy on neurons and satellite glial cells in mouse trigeminal ganglion. Pain 110, 290-298.

Christie, K., Koshy, D., Cheng, C., Guo, G., Martinez, J.A., Duraikannu, A., Zochodne, D.W., 2015. Intraganglionic interactions between satellite cells and adult sensory neurons. Molecular and cellular neurosciences 67, 1-12.

Colucci-Guyon, E., Portier, M.M., Dunia, I., Paulin, D., Pournin, S., Babinet, C., 1994. Mice lacking vimentin develop and reproduce without an obvious phenotype. Cell 79, 679-694.

de Souza Martins, S.C., Agbulut, O., Diguet, N., Larcher, J.C., Paulsen, B.S., Rehen, S.K., MouraNeto, V., Paulin, D., Li, Z., Xue, Z., 2011. Dynamic expression of synemin isoforms in mouse embryonic stem cells and neural derivatives. BMC Cell Biol 12, 51.

Escurat, M., Djabali, K., Gumpel, M., Gros, F., Portier, M.M., 1990. Differential expression of two neuronal intermediate-filament proteins, peripherin and the low-molecular-mass neurofilament protein (NF-L), during the development of the rat. The Journal of neuroscience : the official journal of the Society for Neuroscience 10, 764-784.

Ferri, G.L., Sabani, A., Abelli, L., Polak, J.M., Dahl, D., Portier, M.M., 1990. Neuronal intermediate filaments in rat dorsal root ganglia: differential distribution of peripherin and neurofilament protein immunoreactivity and effect of capsaicin. Brain Res 515, 331-335.

Fields, R.D., Stevens-Graham, B., 2002. New insights into neuron-glia communication. Science 298, 556-562.

Galmiche, G., Labat, C., Mericskay, M., Aissa, K.A., Blanc, J., Retailleau, K., Bourhim, M., Coletti, D., Loufrani, L., Gao-Li, J., Feil, R., Challande, P., Henrion, D., Decaux, J.F., Regnault, V., Lacolley, P., Li, Z., 2013. Inactivation of serum response factor contributes to decrease vascular muscular tone and arterial stiffness in mice. Circ Res 112, 1035-1045.

Gillen, C., Gleichmann, M., Spreyer, P., Muller, H.W., 1995. Differentially expressed genes after peripheral nerve injury. Journal of neuroscience research 42, 159-171.

Gimenez, Y.R.M., Langa, F., Menet, V., Privat, A., 2000. Comparative anatomy of the cerebellar cortex in mice lacking vimentin, GFAP, and both vimentin and GFAP. Glia 31, 69-83.

Goldstein, M.E., House, S.B., Gainer, H., 1991. NF-L and peripherin immunoreactivities define distinct classes of rat sensory ganglion cells. Journal of neuroscience research 30, 92-104.

Griffin, J.W., Hoffman, P.N., 1993. Degeneration and regeneration in the peripheric nervous system. In Peripheral Neuropathy. Edited by Dyck P.J., Thomas P.K. Third edition. W.B. Saunders, Philadelphia, pp. 361-376.

Hanani, M., 2005. Satellite glial cells in sensory ganglia: from form to function. Brain Res Brain Res $\operatorname{Rev} 48,457-476$. 
Hoffman, P.N., Cleveland, D.W., 1988. Neurofilament and tubulin expression recapitulates the developmental program during axonal regeneration: induction of a specific beta-tubulin isotype. Proceedings of the National Academy of Sciences of the United States of America 85, 4530-4533.

Izmiryan, A., Franco, C.A., Paulin, D., Li, Z., Xue, Z., 2009. Synemin isoforms during mouse development: multiplicity of partners in vascular and neuronal systems. Experimental cell research 315, 769-783.

Lariviere, R.C., Julien, J.P., 2004. Functions of intermediate filaments in neuronal development and disease. J Neurobiol 58, 131-148.

Li, Z., Parlakian, A., Coletti, D., Alonso-Martin, S., Hourde, C., Joanne, P., Gao-Li, J., Blanc, J., Ferry, A., Paulin, D., Xue, Z., Agbulut, O., 2014. Synemin acts as a regulator of signalling molecules during skeletal muscle hypertrophy. Journal of cell science 127, 4589-4601.

Liang, L., Wang, Z., Lu, N., Yang, J., Zhang, Y., Zhao, Z., 2010. Involvement of nerve injury and activation of peripheral glial cells in tetanic sciatic stimulation-induced persistent pain in rats. Journal of neuroscience research 88, 2899-2910.

Liu, F.Y., Sun, Y.N., Wang, F.T., Li, Q., Su, L., Zhao, Z.F., Meng, X.L., Zhao, H., Wu, X., Sun, Q., Xing, G.G., Wan, Y., 2012. Activation of satellite glial cells in lumbar dorsal root ganglia contributes to neuropathic pain after spinal nerve ligation. Brain Res 1427, 65-77.

McKay Hart, A., Brannstrom, T., Wiberg, M., Terenghi, G., 2002. Primary sensory neurons and satellite cells after peripheral axotomy in the adult rat: timecourse of cell death and elimination. Exp Brain Res 142, 308-318.

Menet, V., Gimenez y Ribotta, M., Chauvet, N., Drian, M.J., Lannoy, J., Colucci-Guyon, E., Privat, A., 2001. Inactivation of the glial fibrillary acidic protein gene, but not that of vimentin, improves neuronal survival and neurite growth by modifying adhesion molecule expression. The Journal of neuroscience : the official journal of the Society for Neuroscience 21, 6147-6158.

Menet, V., Prieto, M., Privat, A., Gimenez y Ribotta, M., 2003. Axonal plasticity and functional recovery after spinal cord injury in mice deficient in both glial fibrillary acidic protein and vimentin genes. Proceedings of the National Academy of Sciences of the United States of America 100, 89999004.

Nascimento, R.S., Santiago, M.F., Marques, S.A., Allodi, S., Martinez, A.M., 2008. Diversity among satellite glial cells in dorsal root ganglia of the rat. Braz J Med Biol Res 41, 1011-1017.

Nothias, F., Boyne, L., Murray, M., Tessler, A., Fischer, I., 1995. The expression and distribution of tau proteins and messenger RNA in rat dorsal root ganglion neurons during development and regeneration. Neuroscience 66, 707-719.

Oblinger, M.M., Lasek, R.J., 1988. Axotomy-induced alterations in the synthesis and transport of neurofilaments and microtubules in dorsal root ganglion cells. The Journal of neuroscience : the official journal of the Society for Neuroscience 8, 1747-1758.

Pannese, E., 1964. Number and Structure of Perisomatic Satellite Cells of Spinal Ganglia under Normal Conditions or during Axon Regeneration and Neuronal Hypertrophy. Z Zellforsch Mikrosk Anat 63, 568-592.

Pannese, E., 2010. The structure of the perineuronal sheath of satellite glial cells (SGCs) in sensory ganglia. Neuron Glia Biol 6, 3-10.

Pekny, M., 2001. Astrocytic intermediate filaments: lessons from GFAP and vimentin knock-out mice. Progress in brain research 132, 23-30.

Pekny, M., Johansson, C.B., Eliasson, C., Stakeberg, J., Wallen, A., Perlmann, T., Lendahl, U., Betsholtz, C., Berthold, C.H., Frisen, J., 1999. Abnormal reaction to central nervous system injury in mice lacking glial fibrillary acidic protein and vimentin. The Journal of cell biology 145, 503-514.

Perlson, E., Hanz, S., Ben-Yaakov, K., Segal-Ruder, Y., Seger, R., Fainzilber, M., 2005. Vimentindependent spatial translocation of an activated MAP kinase in injured nerve. Neuron 45, 715-726. 
Potokar, M., Kreft, M., Li, L., Daniel Andersson, J., Pangrsic, T., Chowdhury, H.H., Pekny, M., Zorec, R., 2007. Cytoskeleton and vesicle mobility in astrocytes. Traffic 8, 12-20.

Raginov, I.S., Chelyshev Iu, A., 2003. [Post-traumatic survival in different subpopulations of sensory neurons]. Morfologiia 124, 47-50.

Ramer, M.S., Priestley, J.V., McMahon, S.B., 2000. Functional regeneration of sensory axons into the adult spinal cord. Nature 403, 312-316.

Reid, A.J., Welin, D., Wiberg, M., Terenghi, G., Novikov, L.N., 2010. Peripherin and ATF3 genes are differentially regulated in regenerating and non-regenerating primary sensory neurons. Brain Res 1310, $1-7$.

Shinder, V., Amir, R., Devor, M., 1998. Cross-excitation in dorsal root ganglia does not depend on close cell-to-cell apposition. Neuroreport 9, 3997-4000.

Sommer, E.W., Kazimierczak, J., Droz, B., 1985. Neuronal subpopulations in the dorsal root ganglion of the mouse as characterized by combination of ultrastructural and cytochemical features. Brain Res 346, 310-326.

Sugimura, K., Haimoto, H., Nagura, H., Kato, K., Takahashi, A., 1989. Immunohistochemical differential distribution of S-100 alpha and S-100 beta in the peripheral nervous system of the rat. Muscle Nerve 12, 929-935.

Titeux, M., Brocheriou, V., Xue, Z., Gao, J., Pellissier, J.F., Guicheney, P., Paulin, D., Li, Z., 2001. Human synemin gene generates splice variants encoding two distinct intermediate filament proteins. European journal of biochemistry / FEBS 268, 6435-6449.

Vega, J.A., Rodriguez, C., Medina, M., del Valle-Soto, M.E., Hernandez, L.C., 1989. Expression of cytoskeletal proteins in glial cells of dorsal root ganglia. Cell Mol Biol 35, 635-641.

Vergara, D., Romano, A., Stanca, E., La Pesa, V., Aloisi, L., De Domenico, S., Franck, J., Cicalini, I., Giudetti, A., Storelli, E., Pieragostino, D., Fournier, I., Sannino, A., Salzet, M., Cerri, F., Quattrini, A., Maffia, M., 2018. Proteomic expression profile of injured rat peripheral nerves revealed biological networks and processes associated with nerve regeneration. J Cell Physiol 233, 6207-6223.

Watkins, L.R., Maier, S.F., 2002. Beyond neurons: evidence that immune and glial cells contribute to pathological pain states. Physiol Rev 82, 981-1011.

Willis, D., Li, K.W., Zheng, J.Q., Chang, J.H., Smit, A.B., Kelly, T., Merianda, T.T., Sylvester, J., van Minnen, J., Twiss, J.L., 2005. Differential transport and local translation of cytoskeletal, injuryresponse, and neurodegeneration protein mRNAs in axons. The Journal of neuroscience : the official journal of the Society for Neuroscience 25, 778-791.

Wong, J., Oblinger, M.M., 1990. Differential regulation of peripherin and neurofilament gene expression in regenerating rat DRG neurons. Journal of neuroscience research 27, 332-341.

Woodham, P., Anderson, P.N., Nadim, W., Turmaine, M., 1989. Satellite cells surrounding axotomised rat dorsal root ganglion cells increase expression of a GFAP-like protein. Neurosci Lett 98, 8-12.

Xue, Z.G., Cheraud, Y., Brocheriou, V., Izmiryan, A., Titeux, M., Paulin, D., Li, Z., 2004. The mouse synemin gene encodes three intermediate filament proteins generated by alternative exon usage and different open reading frames. Experimental cell research 298, 431-444. 
SynM/NFL
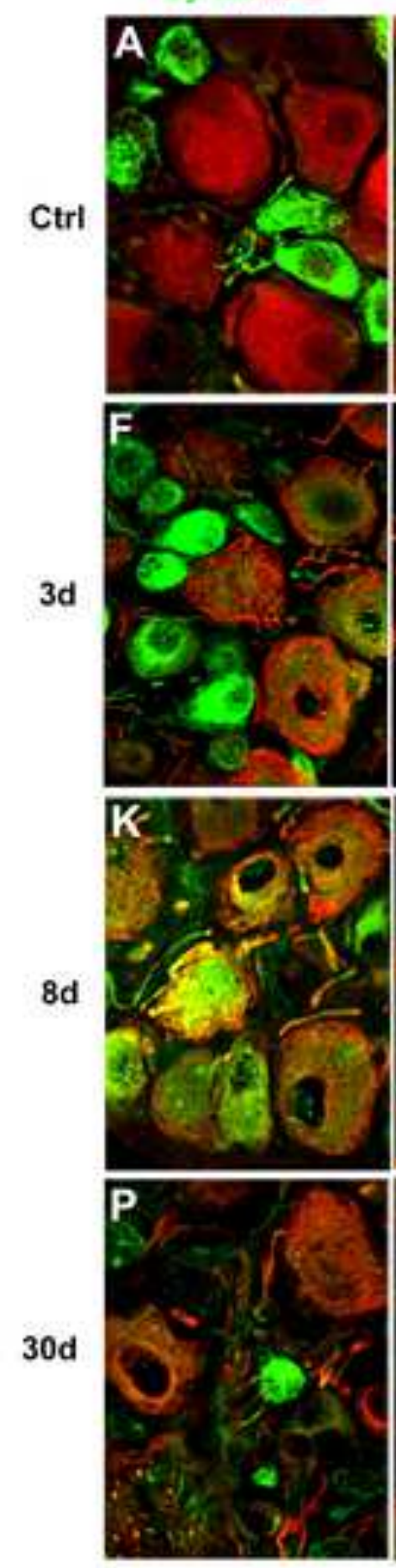

U

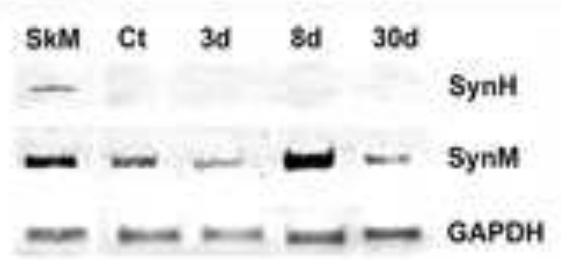

SynLINFL
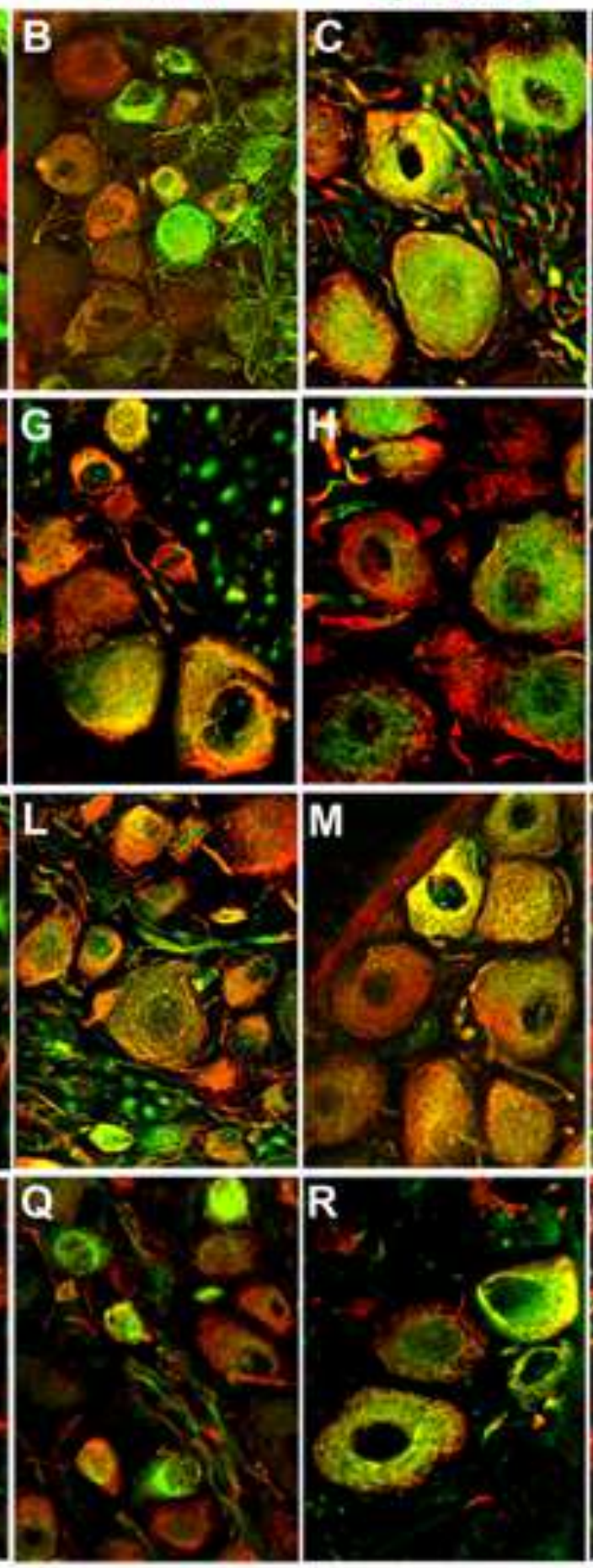

V
SynL/Per
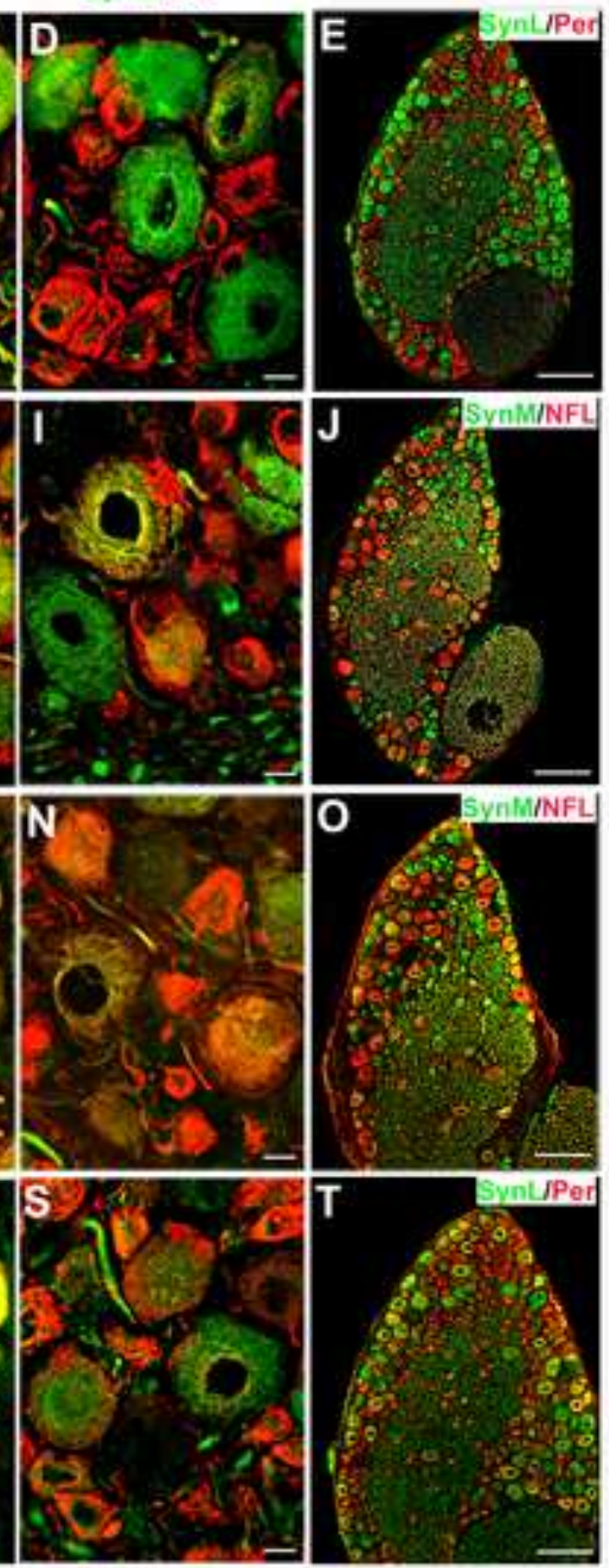

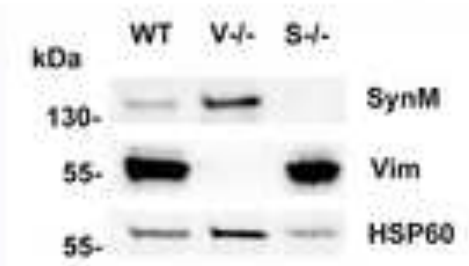



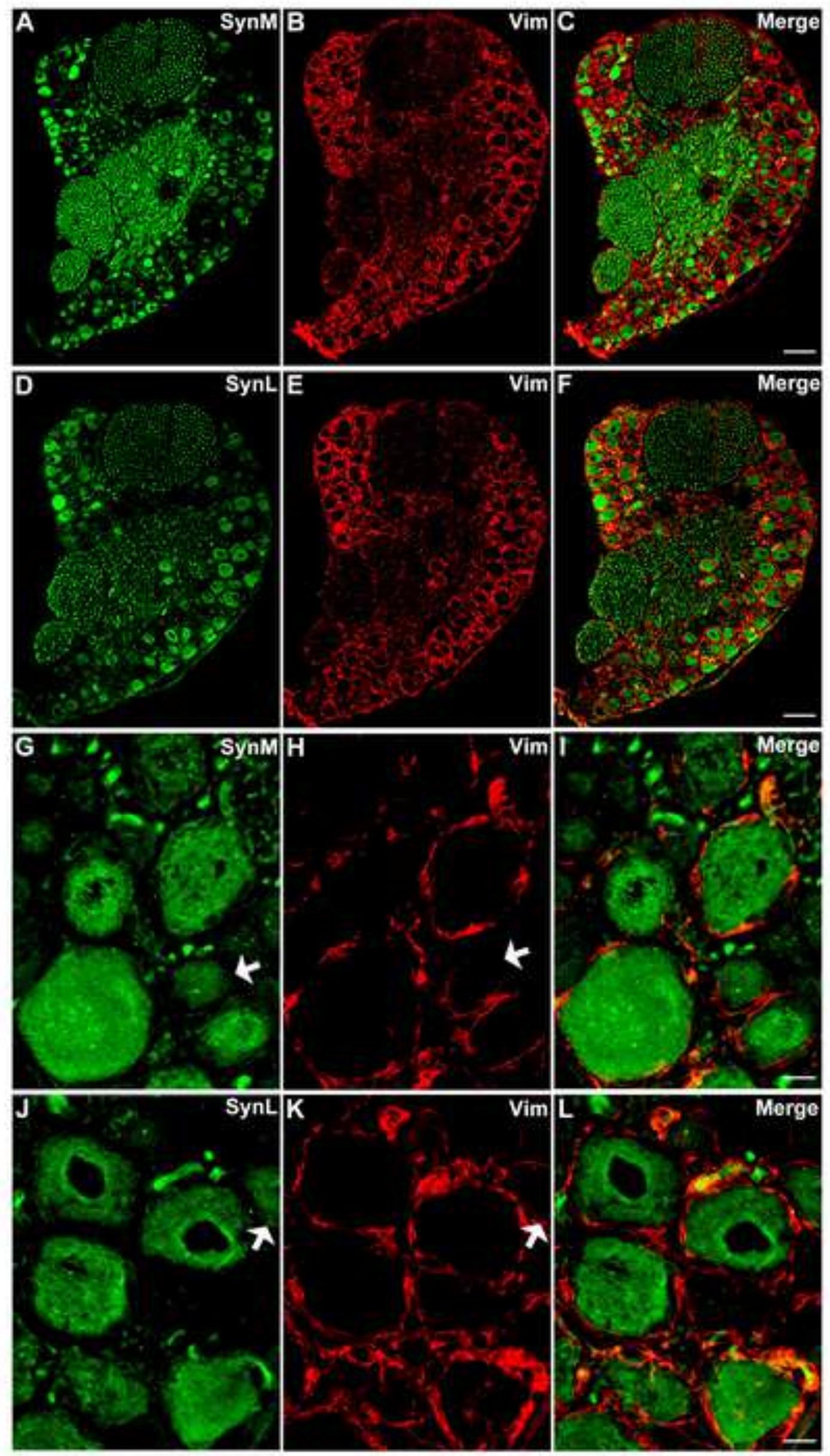

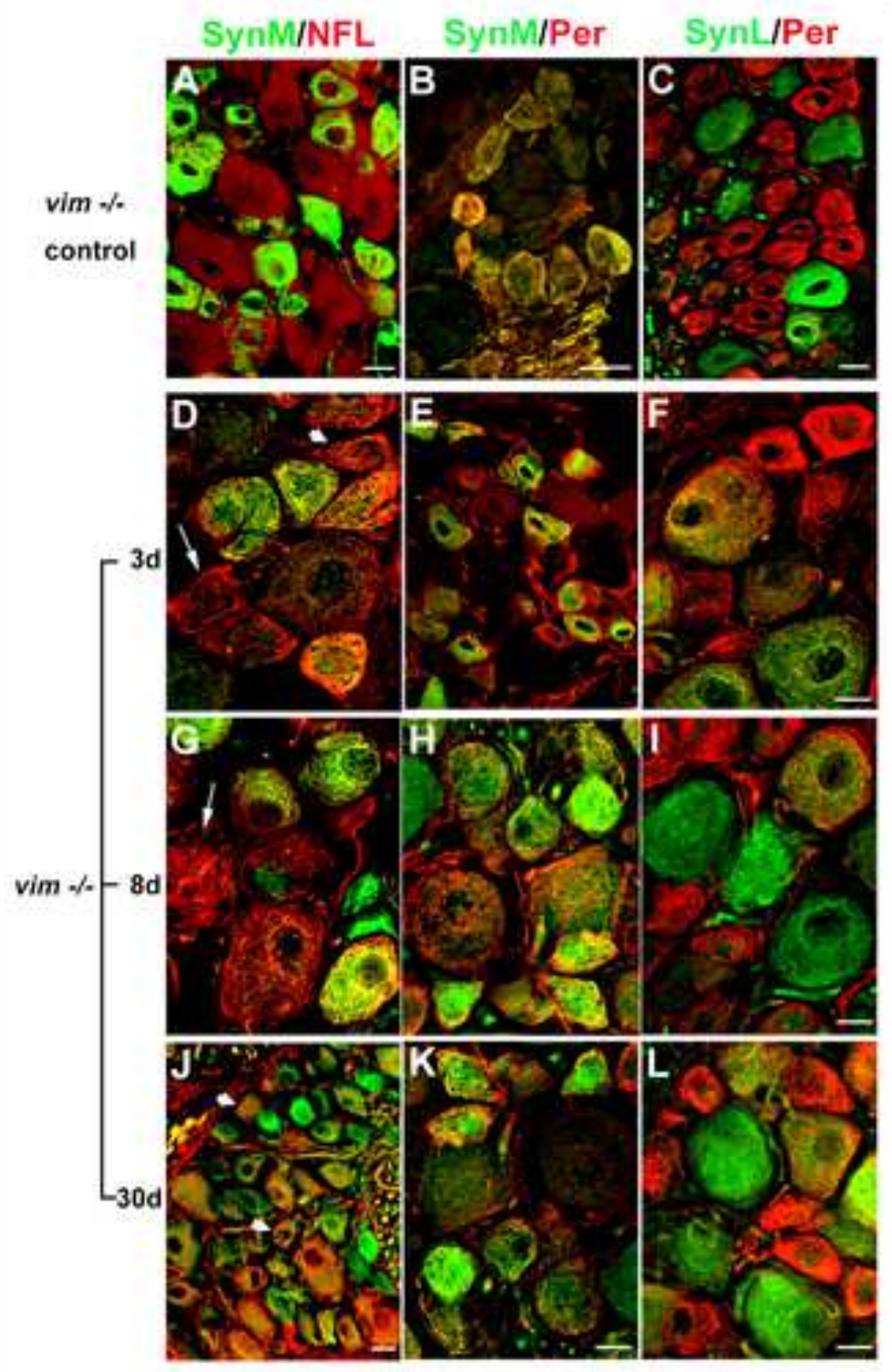

synm- control
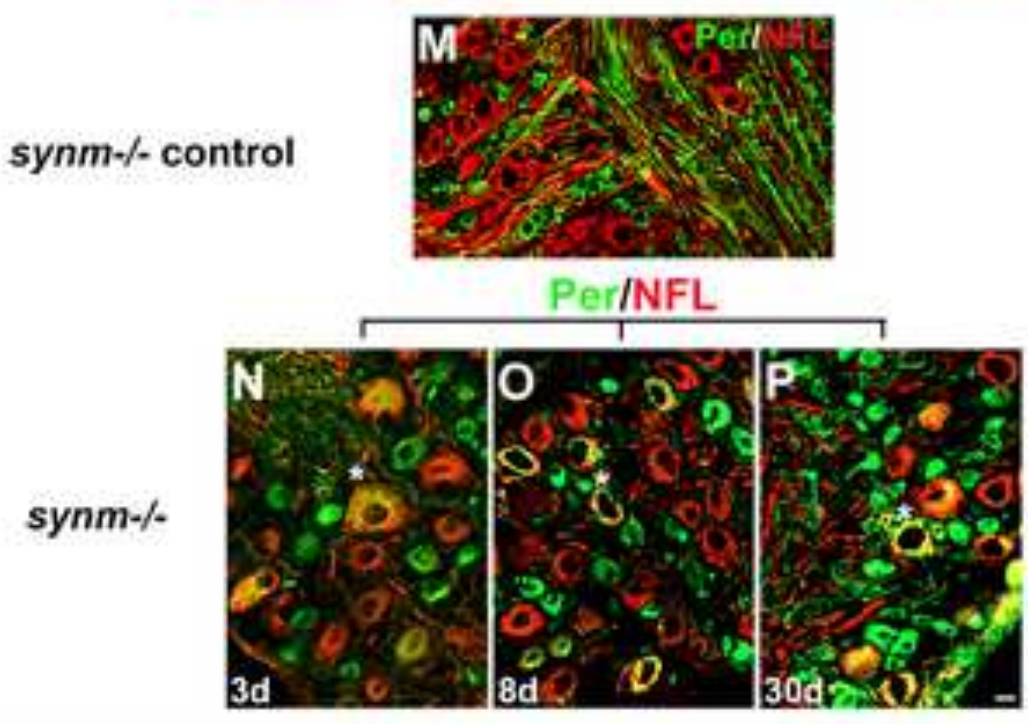

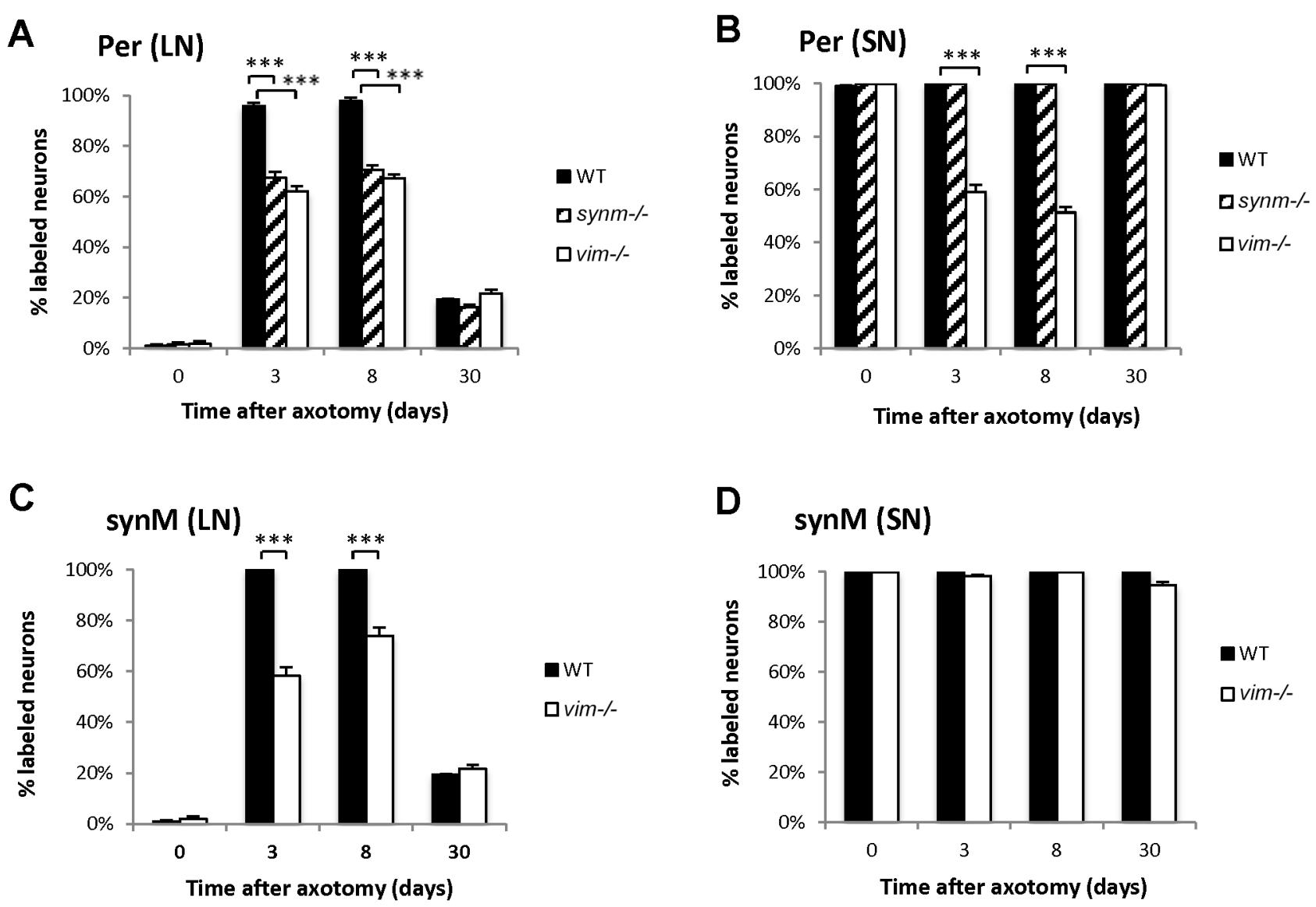

E

$$
\text { NFL (LN) }
$$
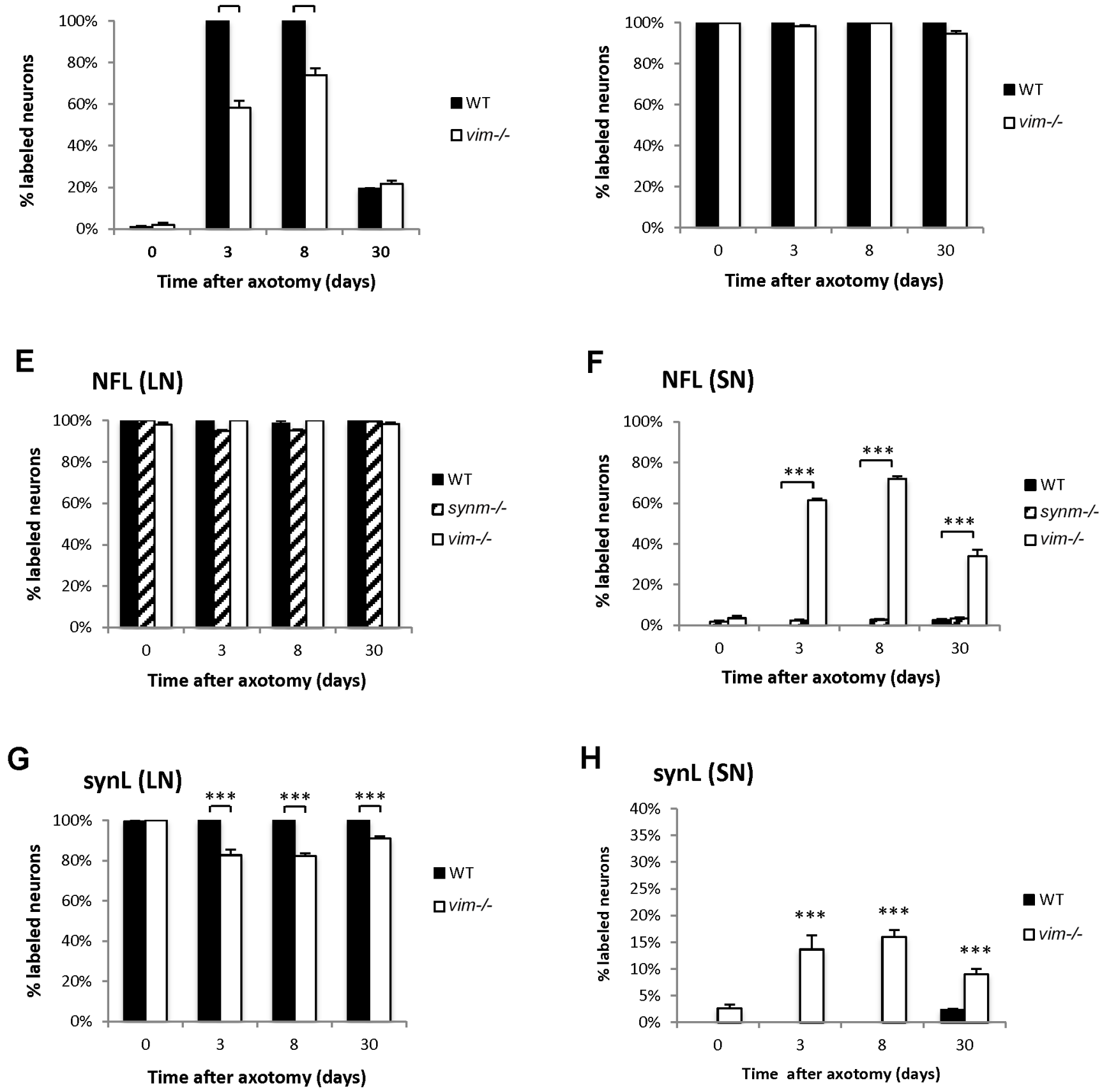

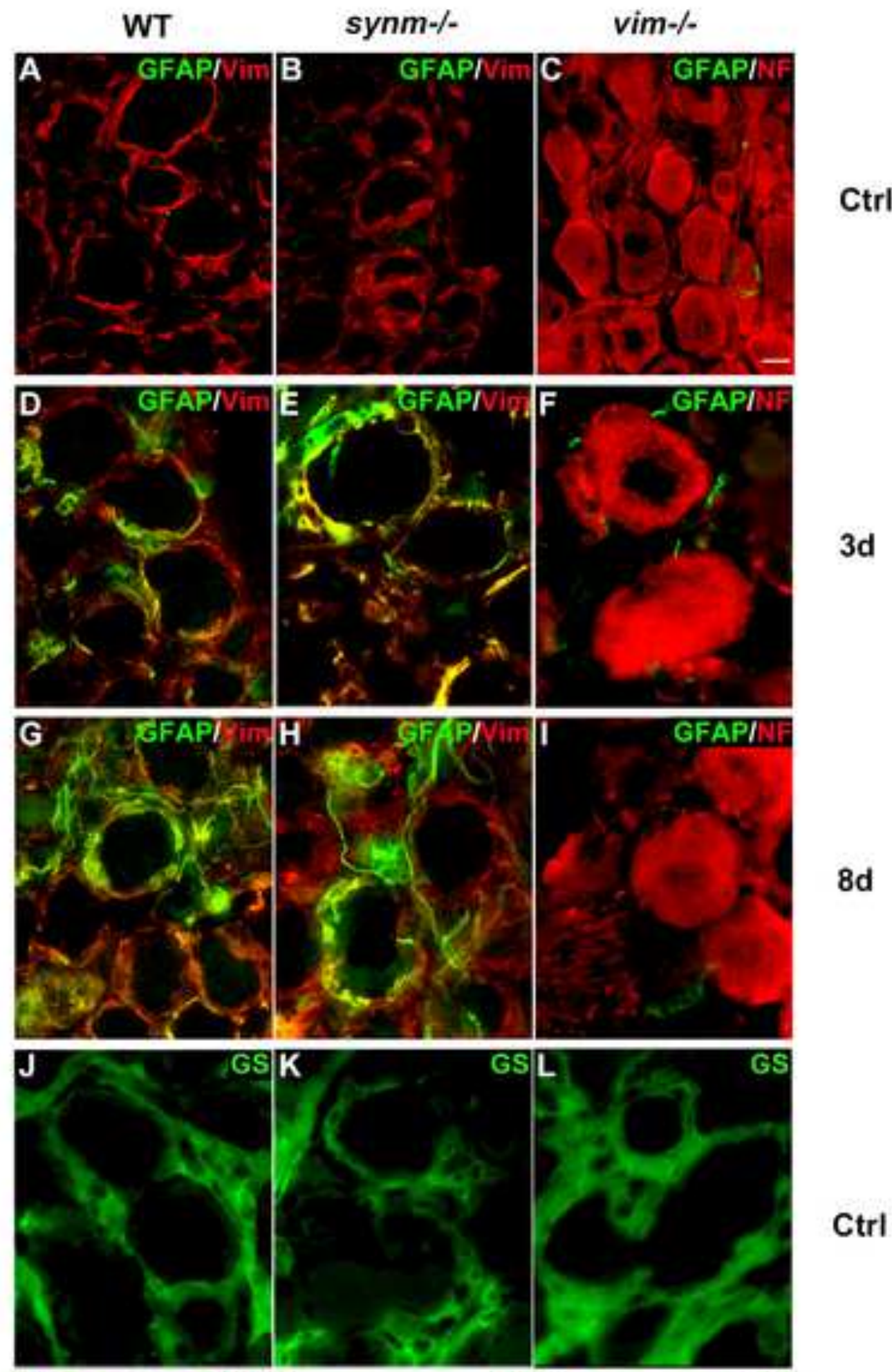

Ctrl

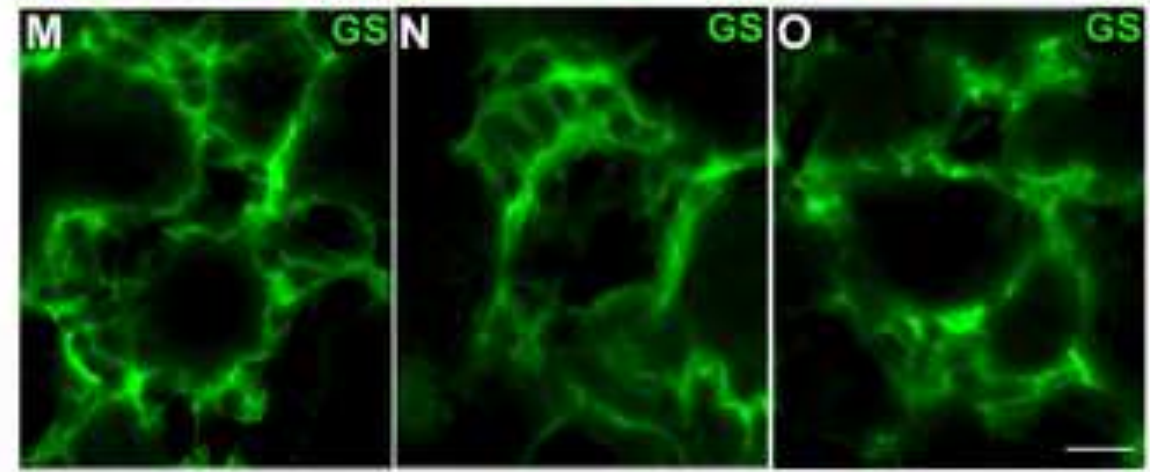

3d 
A
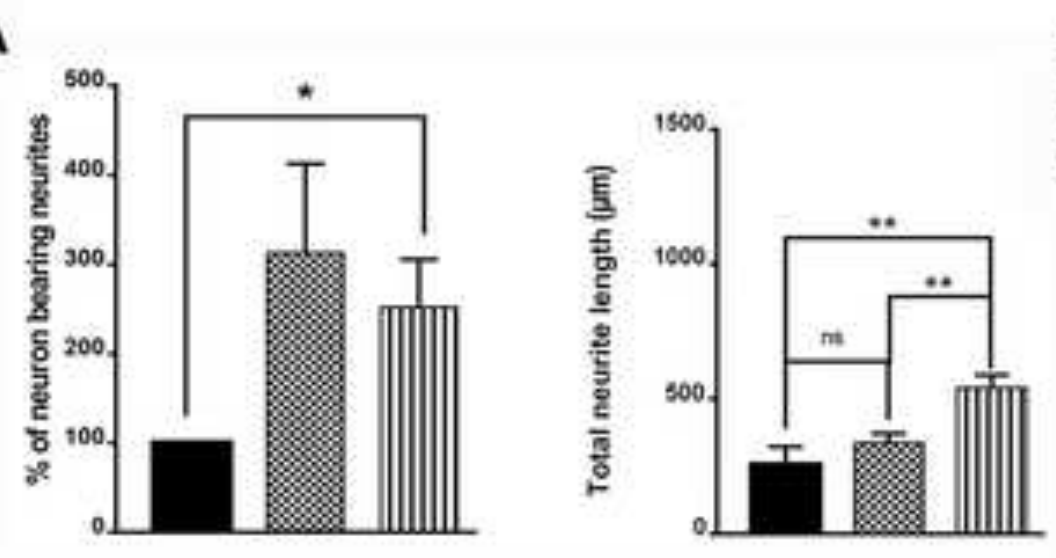

C

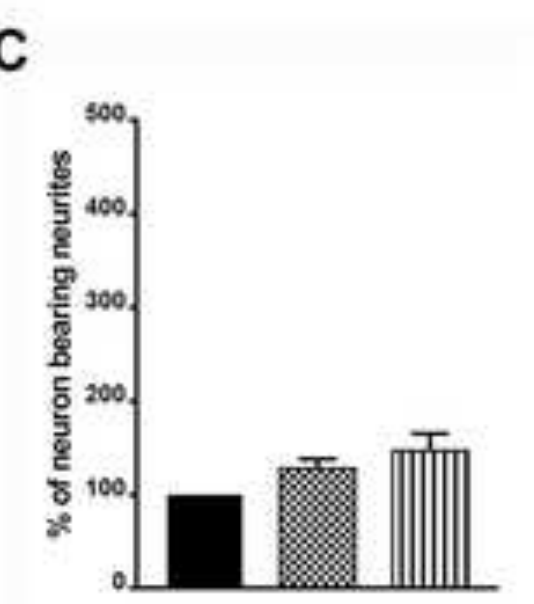

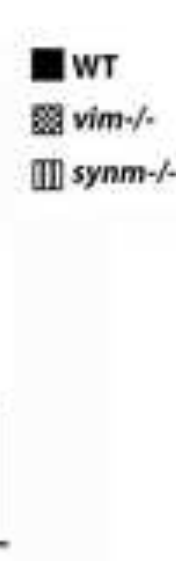

B

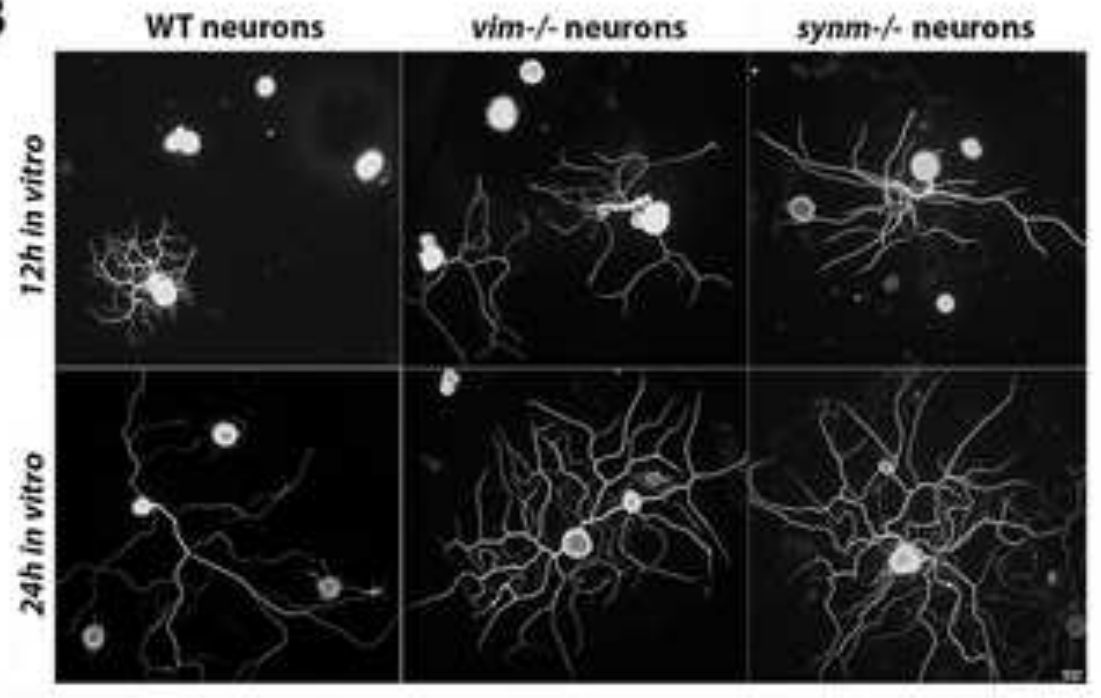

D
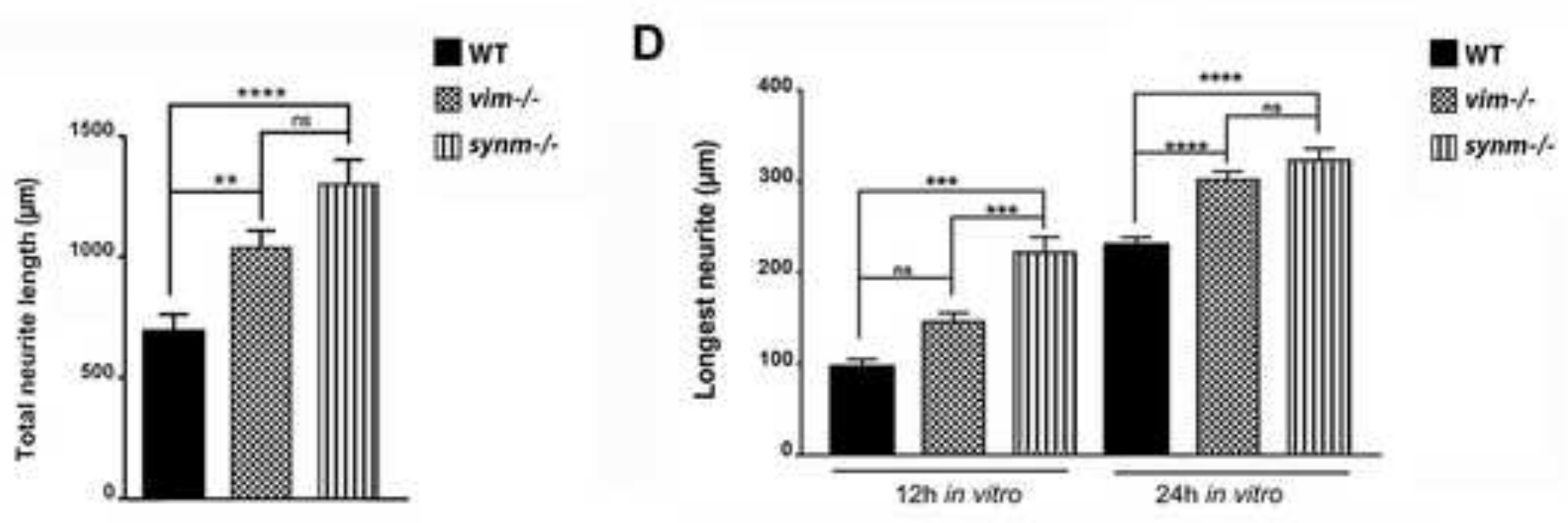

aT

雨 vim $\%$

III) synm $\%$ - 